\title{
El Centro de Cardioestimuladores del Uruguay'. CCC Medical Devices
}

\author{
Darscht, P. ${ }^{(1,2)}$ \\ Contacto: pdarscht@gmail.com \\ (1) Consultor de INGENIO - Laboratorio Tecnológico del Uruguay (LATU) - (2) Programa PACPYMES
}

\begin{abstract}
Resumen
Estudio de caso del Centro de Cardioestimuladores del Uruguay - CCC Medical Devices preparado a solicitud de Ingenio en el marco del proyecto financiado por la Iniciativa para Incubadoras de InfoDev - Grupo Banco Mundial.

Este estudio detalla los pasos seguidos por una empresa nacional con un fuerte factor de innovación y los cambios producidos en el entorno de los negocios de la empresa. El comienzo de una pequeña empresa de marcapasos que tras pasar por diferentes etapas hoy gana mercados en el área de ingeniería para dispositivos médicos para diferentes empresas de investigación biomédica a nivel internacional.

\section{Summary}

Case study of the Centro de Cardioestimuladores del Uruguay - CCC Medical Devices prepared on behalf of Ingenio within the project financed by the Incubator Initiative of InfoDev - World Bank Group.

This study refers to the steps followed by a highly innovative local company and to the changes in its business environment. The start up of a small pacemakers company that after going through different stages is presently increasing its market share in the area of engineering of medical devices for biomedic research companies worldwide.
\end{abstract}

\begin{abstract}
$\mathrm{A}^{\mathrm{f}}$ fines de 2004, el Gerente de Negocios Internacionales de CCC Medical Devices, Ingeniero Fernando Brum, narra el más reciente cambio en el entorno de negocios de la empresa:

A principios del año pasado, nosotros habíamos tomado la decisión de poner mucho fuego bajo el tema marcapasos y bajo el tema productos propios. No obstante, las noticias de los últimos dos meses indican un incremento de la demanda por fabricación de dispositivos que nuestra División Ingeniería ha venido diseñando para nuestros clientes de outsourcing. Nos está pasando que no podemos salir a buscar nuevos mercados de marcapasos, porque ya negociamos para empezar a vender marcapasos a Brasil en 2005. Es decir, si en marzo comenzamos a vender en Brasil, si conseguimos algún otro cliente de acá del medio, vamos a tener un lío espantoso de producción, no vamos a poder estar entregando.

En estas páginas se reporta la particularísima historia de esta empresa, plagada de "bandazos" que escandalizarían a cualquier planificador ortodoxo. Pero a poco que se examina con algo de cuidado, se identifica un invariante que caracteriza cada una de sus fases de desarrollo: la nueva fase no hubiera sido posible, sin las capacidades generadas en las fases anteriores. En efecto, el Dr. Fiandra instaló una pequeña empresa para fabricar marcapasos porque había participado del desarrollo de esa tecnología de punta en Suecia, conocía por tanto la tecnología y había realizado los primeros implantes exitosos en humanos de estos dispositivos. CCC pudo luego ensamblar marcapasos de terceros, porque conocía del mercado de estos dispositivos médicos y tenía una marca reconocida localmente. Pudo, años más tarde, volver a diseñar y fabricar sus propios dispositivos debido a que permaneció en el mercado y mantuvo su capacidad empresarial, de producción y de marketing. Ingresó más tarde en el negocio global de los servicios de ingeniería biomédica, porque sus productos, diseñados por su departamento de ingeniería, daban fe de una experiencia y calidad reconocida. Hoy gana mercados en el área de los marcapasos, porque se sabe que su ingeniería ha sido contratada por prestigiosas empresas de investigación biomédica, las que a su vez, le están
\end{abstract}

encargando la fabricación de dispositivos implantables que la propia CCC diseñó.

\section{El Comienzo}

El Centro de Cardioestimuladores del Uruguay S.A., CCC, fue fundado en 1969 con el objetivo de abastecer el mercado doméstico de cardioestimuladores, en ese entonces un dispositivo muy costoso y de difícil acceso desde el Uruguay, fabricado en Estados Unidos y Suecia. Uno de sus fundadores, el Dr. Fiandra, había participado en Suecia del grupo de desarrollo que logró el primer marcapasos exitoso, que fue implantado precisamente por el Dr. Fiandra, junto al Dr. Rubio, el 3 de febrero en 1960 en Uruguay. Cuenta el Dr. Fiandra:

Yo me acuerdo que un colega que se dedicaba a la especialidad, muy amigo, me dijo: "pero vas a ponerte a fabricar algo así, es una idea loca, para implantar 3 ó 4 por año, 5 por año.."

Esa "idea loca" dio origen a una empresa que, 35 años después, no solo sigue fabricando cardioestimuladores en Uruguay para el mundo, sino que ha utilizado las capacidades que fue generando para mantenerse competitiva en ese mercado para ingresar exitosamente en un negocio más ambicioso aún, cuyos clientes son los equipos de investigación biomédica de institutos científicos de renombre mundial: el diseño y fabricación a medida de dispositivos implantables.

Inmediatamente, surgen una serie casi interminable de preguntas: ¿Cómo pudo ser posible esto en Uruguay? ¿Cómo hizo CCC para vender los primeros cardioestimuladores en el exterior? ¿Cómo puede mantenerse competitiva vendiendo un producto que, uno supone, hoy en día es casi un "commodity sofisticado"?. Y más relevante aún, ¿cómo consiguió pasar de ser un fabricante de ese "commodity sofisticado" a ser un proveedor tecnológico de clientes extremadamente exigentes? ¿es sustentable su estrategia? ¿Qué lecciones pueden extraer de la misma otras empresas del área tecnológica que quieren acceder a los mercados internacionales?

1 Este caso de estudio ha sido preparado por Pablo Darscht para la Incubadora INGENIO. La información que se presenta no necesariamente refleja la opinión de CCC. El objetivo del mismo es motivar la discusión de un caso real con objetivos de aprendizaje más que ilustrar el manejo efectivo o inefectivo de una situación gerencial. 


\section{Los Marcapasos}

Un marcapasos es un dispositivo electrónico usado en la mayoría de los casos como un implante permanente, que regula el ritmo del latido cardiaco. Se utiliza para producir un ritmo cardiaco normal en pacientes con un ritmo anormalmente bajo (bloqueo cardiaco). Actualmente es un pequeño aparato plano de unos 5 por $3 \mathrm{~cm}$ y un peso de 25-40 g. Posee una batería de litio-yodo con una vida de hasta 10 años. Se implanta bajo la piel del pecho y se inserta un electrodo aislado en una gran vena que entra en la parte derecha del corazón. Cada latido eléctrico pasa por ese electrodo hasta el músculo cardiaco, obligándolo a latir (contraerse).

Durante los años 60 se realizaron pocos implantes en Uruguay, debido a los costos extremadamente altos de los dispositivos importados. En ese contexto, el Dr. Fiandra crea CCC para abastecer el mercado doméstico y lograr una baja importante en los precios y facilitar el acceso a productos de esa tecnología.

Durante los años siguientes, CCC diseñó y fabricó estos equipos, que eran bastante primitivos, consistiendo básicamente en un timer y un generador de pulsos. CCC fue el cuarto fabricante a nivel mundial en utilizar energía nuclear en sus marcapasos, y el sexto en usar baterías de litio, que hoy se han convertido en la fuente estándar de energía para este tipo de dispositivos.

En los ochenta, la tecnología de los marcapasos maduró, incorporándose funciones que permitían medir determinados parámetros de las señales y comenzaron a aparecer los primeros modelos programables. La tecnología que hacía posible estas funciones requerían una inversión bastante alta para cada modelo y nuevas técnicas de diseño electrónico, no disponibles localmente. Dado el reducido tamaño del mercado interno, y la voluntad de empresas americanas de licenciar sus diseños a terceras empresas para que actuaran como ensambladoras, CCC opta por realizar un acuerdo para ensamblar para el mercado local los marcapasos de una empresa de Estados Unidos.

A principios de los 90 ocurrió un nuevo salto tecnológico, que consistió en la incorporación de microcomputadores adentro de los marcapasos ${ }^{2}$. Esto cambió, una vez más, las reglas del negocio, y por lo tanto motivó un replanteo estratégico de CCC. Por un lado, desaparecieron las empresas medianas o grandes que licenciaban tecnología a ensambladores. Por otra parte, el proveedor de tecnología estadounidense de CCC intentó acompañar el cambio tecnológico e introdujo un nuevo modelo de cardioestimulador programable, que no terminaba de funcionar a satisfacción. Por su parte, CCC adviertió rápidamente que una consecuencia de la nueva tecnología disponible era una disminución sustancial de los costos de diseño de nuevos productos, ya que el "cerebro" de los mismos podía ser fabricado en base a componentes estándar (microcontroladores disponibles comercialmente como circuitos integrados de propósito general). En otras palabras, un marcapasos pasó a consistir en un conjunto de circuitería electrónica comandada por una computadora que tiene adentro. Esta tecnología, a diferencia de la anterior, tenía barreras a la entrada muy bajas. Por otra parte, era esencialmente la misma que se aplicaba desde hacía ya varios años en Uruguay en áreas como la automatización industrial y las telecomunicaciones; consecuentemente, se disponía localmente de recursos humanos capacitados para aplicarla. Otra característica no menor de la "nueva" tecnología era que una serie de problemas que hasta ese entonces debían ser resueltos por hardware (es decir, con circuitería electrónica compleja y a medida) pasaban a poder ser resueltos escribiendo un programa apropiado para el microcontrolador.
En ese escenario, la empresa decidió constituir un equipo, integrado por dos ingenieros electrónicos y dos informáticos, para desarrollar sus propios cardioestimuladores basados en esta nueva tecnología. Este proyecto resultó exitoso, y la empresa volvió a diseñar y fabricar marcapasos localmente.

En Uruguay, la implantación de los marcapasos es financiada por el "Fondo Nacional de Recursos" (FNR), una entidad estatal que solventa determinados tratamientos médicos considerados "altamente especializados". Toda persona tiene acceso a estos tratamientos, independientemente de que su cobertura de salud ocurra a través de una institución de asistencia médica colectiva (atención mutual privada) o por intermedio de Salud Pública (atención gratuita a personas de bajos recursos). Según un reporte de mediados de 2003, el FNR había utilizado 5 marcas de marcapasos, 4 de ellas importadas, distribuyéndose su uso de la siguiente forma:

\begin{tabular}{|c|c|}
\hline Marca A & $10.3 \%$ \\
\hline Marca B & $13.9 \%$ \\
\hline Marca C & $19.0 \%$ \\
\hline Marca D & $27.0 \%$ \\
\hline CCC & $29.6 \%$ \\
\hline
\end{tabular}

Como se observa, los marcapasos CCC son los más utilizados en el país. En el último año reportado, se colocaron 462 marcapasos CCC. De ellos 117 correspondieron a pacientes de Salud Pública y 345 a pacientes provenientes del sector privado. Es decir que el $75 \%$ de los marcapasos CCC fueron implantados en pacientes provenientes del sector privado. ${ }^{3}$

\section{Ayudar a latir fuera de Uruguay}

Los primeros intentos de vender los productos de CCC en el exterior fueron realizados por médicos conocidos del Dr. Fiandra, que intentaron introducir los marcapasos uruguayos en sus respectivos países. Estos intentos fracasaron, al carecer sus impulsores de experiencia en temas empresariales específicos. Cuando recuerda el primer intento de salir al exterior, Brum afirma que "fue desastroso", y prosigue:

Era el año 1995 y decidimos presentarnos en el congreso mundial de marcapasos que se hacía en Buenos Aires. Llegamos con un producto que considerábamos maravilloso, muy adecuado para el mercado y que estaba diseñado específicamente para países del Tercer Mundo... el problema fue que el mercado no tenía el más mínimo interés en él.

Analizando esa experiencia, los directivos de CCC llegaron a la conclusión que la primera cuestión central a definir era cuál debía ser, en realidad, su negocio. Se trataba de una empresa integrada y dirigida por un grupo de ingenieros y médicos que entendían de tecnología, pero no de marketing, canales de distribución y servicio al cliente. A los efectos de alinear la definición del negocio con las capacidades de la empresa, surge la idea de convertirse en un proveedor global de servicios de ingeniería altamente especializados, que se referirá más adelante. De todas maneras, la venta de marcapasos al mundo no ha sido descuidada por CCC. Por el contrario, se han ido explorando diversas estrategias de negocios, con diverso grado de éxito.

Funciona aceptablemente una alianza con un trader (propiedad de un ruso) radicado en Estados Unidos, que atiende el mercado

2 Un característica de la evolución tecnológica de los dispositivos con requerimientos muy exigentes en materia de confiabilidad, es que la incorporación de avances se produce recién cuando la "nueva" tecnología ya ha ingresado en la fase de madurez. Este fenómeno se observa sistemáticamente en el caso de los dispositivos implantables. A modo de ejemplo, obsérvese que el primer microprocesador, el Intel 4004, comenzó a ser fabricado en 1971; en los '80 los microprocesadores y microcontroladores constituían ya una tecnología fácilmente accesible y de bajo costo, que se utilizaba sistemáticamente para incorporar masivamente "inteligencia” a los equipos electrónicos que se fabricaban en todo el mundo, incluyendo, por supuesto, el Uruguay. No obstante, la tecnología de microcontroladores recién sería incorporada a los marcapasos en la década de 1990.

3 Fuente: Comunicado del FNR “Sobre la adjudicación de la licitación para la compra de Marcapasos”, noviembre 2003. 
de Rusia. La firma uruguaya les provee de circuitos electrónicos y software y la firma rusa los ensambla y los vende con su marca. Existen una serie de distribuidores muy chicos en mercados específicos, que nunca terminan de crecer. En algunos países, tales como Grecia, se dispone de un buen distribuidor, pero la falta de la marca CE (ver anexo 1) es un impedimento fundamental para su desarrollo. Surgieron buenos distribuidores en Argentina (donde el proceso fue particularmente trabajoso debido a la oposición de los importadores de las marcas grandes), Ecuador, República Dominicana, entre otros.

A nivel internacional, los productos de CCC compiten por precio en la franja intermedia. No ofrece los modelos ultra-sofisticados que serían necesarios para ingresar al mercado norteamericano, el que por otra parte tiene otras importantes barreras a la entrada, como lo son los costos de los seguros que es necesario contratar. En Europa, en cambio, podría ingresarse al mercado si se dispusiera de la certificación CE, proceso en el que se haya embarcado CCC junto a un socio europeo, y espera culminar en 2005.

\section{Un proveedor de ingeniería}

A mediados de los 90, CCC había desarrollado capacidades tecnológicas importantes en el área de diseño y fabricación de dispositivos implantables, no tenía mayores posibilidades de crecimiento en el diminuto mercado local, y enfrentaba las dificultades propias de comercializar internacionalmente un producto relativamente "estándar" producido en un país cuyos costos relativos eran altos y su imagen tecnológica inexistente. La empresa estaba muy orientada a la tecnología, y tenía carencias en el proceso de distribución, debidas sobre todo a la magnitud de los recursos que se necesitan para comercializar a nivel mundial un producto de las características de los cardioestimuladores. Ya con varias décadas de presencia en el mercado, la empresa tenía una idea clara de cómo se compone la cadena de valor de su industria, y del papel que podía jugar $\mathrm{CCC}$ en ese contexto : $^{4}$

En la cadena de la tecnología está por un lado la ciencia básica en los orígenes, y hacia el final está la colocación de los productos en el mercado. En esa larga cadena en que de ciencia básica pasamos a ciencia aplicada, a primeras expresiones tecnológicas, a prototipos, a productos y luego a distribución, estamos ubicados en el medio, brindamos servicios a empresas de investigación que nos dicen "Queremos un equipamiento que haga tal y tal cosa".

Esta nueva visión del negocio permitía evitar una estrategia de internacionalización basada en la comercialización de sus productos finales, que presentaba los inconvenientes ya reseñados, y en cambio se apoyaba fuertemente en las capacidades tecnológicas que había desarrollado CCC para llegar a disponer de dichos productos. Ello les permitía mostrar una experiencia fácilmente comprobable en el área de fabricación y diseño.

Así definido el negocio, los clientes son empresas de investigación, básicamente en Estados Unidos, Israel y alguna en Europa, que solicitan un desarrollo de ingeniería. Un ejemplo importante es la empresa estadounidense Impulse Dynamics, que desarrolló una nueva terapia para el tratamiento de la insuficiencia cardíaca, encomendándole a CCC el diseño de un equipo especializado ${ }^{5}$ :

Incluso en una primera instancia ni siquiera sabíamos qué hacía el equipo: firmamos un acuerdo de confidencialidad muy fuerte; sabíamos qué tipo de onda eléctrica teníamos que generar, en qué momento, pero no dónde iban colocados los cables en el implante, ni teníamos conocimiento de las patentes que estaban protegiendo esa investigación. Desarrollamos la primera versión del equipo en 1998, luego varias versiones más; y en este momento, con ese equipo, se está haciendo ensayos clínicos en humanos en Europa, precisamente en Alemania e Italia, y también en Estados Unidos, donde fueron aprobados en 2004. Ya se han realizado los primeros implantes.

Este fue un proceso que nosotros tomamos en 1998. Ellos habían empezado a trabajar antes; pasamos todas las etapas, desde diseñar y fabricar un equipo muy grande, que se conectaba externamente a animales para hacer los experimentos, a tener hoy un equipo implantable, aplicable para uso humano, que se ha empezado a implantar exitosamente en Europa y va a continuar en Estados Unidos el año próximo.

En el caso de la empresa israelí BioControl, CCC vendió a la misma no ya su capacidad de desarrollo y fabricación de un nuevo producto, sino una tecnología "genérica" desarrollada previamente y que utiliza en sus propios productos. En efecto, una característica común de todos los dispositivos implantables programables, es la necesidad de comunicarse con los mismos desde el exterior para intercambiar información en forma bidireccional (ejemplos: modificar parámetros de programación del dispositivo, o "descargar" datos del mismo, como su configuración actual o valores de mediciones efectuadas por el dispositivo).

\section{El primer contrato}

En área de negocios en el que comenzó a incursionar CCC en los 90, a diferencia del "tradicional" de fabricación y venta de cardioestimuladores, es un área de servicios. Por tanto, no existen "muestras" que la empresa pueda enviar al potencial cliente, a los efectos de que éste verifique las cualidades del producto. La reputación juega un papel muy importante. CCC es un jugador conocido en ese ámbito hiperespecífico de la ingeniería para dispositivos implantables, y lo es porque antes otras empresas los contrataron. Por tanto, a la primera pregunta que todo potencial cliente hace cuando contacta a esta empresa uruguaya, es decir, qué han hecho antes y para quién, se le puede responder con una historia de proyectos para grupos de primer nivel internacional ejecutados a satisfacción.

Evidentemente, la primera vez que apareció una oportunidad de comercialización de servicios de ingeniería, la situación era distinta. Si bien existía una trayectoria importante, relativa al diseño y fabricación de cardioestimuladores, CCC no tenía antecedentes para mostrar en el área de ofrecer este servicio a terceros ${ }^{6}$ :

...tomaron contacto con nosotros y nos preguntaron si teníamos algún marcapasos capaz de estimular a alta frecuencia para utilizar en ensayos con perros. En ese momento no teníamos y contestamos que no teníamos porque los marcapasos normales, al contrario, tienen limitada la posibilidad de ir a alta frecuencia, por definición de diseño, pero que lo podíamos hacer, que en un par de semanas podíamos modificar un producto existente y hacerlo.

Nos preguntaron el precio y el plazo; les dimos un muy buen precio y un muy buen plazo. Nos probaron, nos hicieron un pequeño contrato de algunos miles de dólares, luego vinieron a visitarnos. Un factor fundamental en todo este proceso fue que fabricáramos marcapasos, que pudiéramos mostrar una historia de más de veinte años para atrás, con un número enorme de pacientes, varios miles. Podíamos y podemos decir que en nuestra historia tenemos varios miles de pacientes sin inconvenientes.

El precio fue importante para que nos aceptaran la primera prueba: el primer test les salió muy barato; entonces podían darse el lujo de invertir en unos tipos desconocidos que tenían un background de un montón de aparatos implantados. Vinieron a visitarnos, examinaron la fábrica, vieron que tenía estándares de calidad adecuados, que había una historia, un seguimiento, básicamente

4 Fuente: Entrevista realizada al Ingeniero Fernando Brum en el programa "En Perspectiva”, Radio El Espectador, 7/12/01 
referido al mercado local, pero que estaba todo muy prolijamente hecho, y luego tuvimos plazos muy buenos para la entrega.

En esa etapa inicial, tampoco ayudaba el hecho de provenir de un país tan poco nombrado en materia tecnológica. Con el correr de los años, y una vez que la empresa forjó su propia reputación, importó cada vez menos dónde tiene radicada su sede.

A los efectos de cimentar la reputación sobre bases objetivas y permanentes, la dirección de CCC otorga una gran importancia a la política de aseguramiento de la calidad, incluyendo los procesos de certificación internacional. Por ese motivo, la empresa ha sido certificada de acuerdo a las normas ISO9001 y ISO13485 (Ver Anexo 1).

\section{Los Clientes}

Los clientes del departamento de ingeniería de CCC son básicamente empresas que desarrollan investigación.

Su principal cliente es la empresa estadounidense Impulse Dynamics N.V. ${ }^{7}$, focalizada en el desarrollo de terapias eléctricas para el tratamiento de fallas cardíacas, obesidad y diabetes. Su tecnología propietaria está basada en un descubrimiento científico referido al control eléctrico de los tejidos: la aplicación de señales eléctricas no exitatorias a los tejidos puede modificar sus propiedades (por ejemplo, mejorar la contractilidad del músculo cardíaco) en una forma predecible y reproducible. El grupo Impulse Dynamics comenzó en 1996 con el objetivo de investigar y apoyar el desarrollo de esta tecnología propietaria. En su filial estadounidense, en New Jersey, incluye el Centro de Desarrollo de Productos. El Centro de Investigación Tecnológica de Impulse Dynamics está en Israel, y se concentra en las fases tempranas de innovación y desarrollo tecnológico, así como en las tareas experimentales asociadas. La filial europea, Impulse Dynamics $\mathrm{GmbH}$, coordina y asiste en la implementación de pruebas clínicas en ese continente. La empresa lleva adelante programas de investigación en importantes universidades y hospitales de todo el mundo.

Otro cliente es la empresa BioControl Medical LTD, una empresa israelí establecida en 1999, financiada con capital de riesgo, que está desarrollando un dispositivo implantable para la incontinencia urinaria femenina

\section{Los Competidores}

La división ingeniería de CCC compite en el sector de los proveedores de soluciones tecnológicas en materia de dispositivos electrónicos implantables para la industria médica. Así describe la situación de la industria de los dispositivos médicos el reporte anual 2003 de uno de los principales jugadores del sector en que compite CCC, la empresa americana MedSource Technologies:

La tendencia hacia el outsourcing en la industria de dispositivos médicos continuó su crecimiento sólido durante 2003. El mercado actual estimado de servicios de ingeniería y manufactura en nuestros cuatro puntos focales ${ }^{8}$ de la industria médica totaliza actualmente U\$S 3.600 millones y crece a una tasa anual del 13 al $15 \%$. El crecimiento del outsourcing continua siendo guiado por varios factores. En primer lugar, la velocidad de acceso al mercado es cada vez más importante. (...) Segundo, nuestros clientes continúan focalizando sus recursos en investigación sobre nuevas tecnologías de producto y la distribución de los mismos. (...) Tercero, nuestros clientes están experimentando presiones de precios crecientes, debido a que los gastos en salud aumentan a una tasa insostenible, lo que los conduce al establecimiento de relaciones estratégicas con proveedores de servicios que le permitan alcanzar mayor eficiencia y costos más competitivos.

Todos los informes que analizan la industria coinciden, efectivamente, en que el sector crecerá en los próximos años. Por ejemplo, Freedonia Group, una empresa norteamericana dedicada a la investigación de mercados tecnológicos, afirma que la demanda por implantes médicos crecerá, en Estados Unidos, a una tasa de casi el $11 \%$ hasta el año 2007. Como factores principales se mencionan el envejecimiento de la población, los avances de los productos y los cambios en las estrategias de cuidados de los pacientes. Se prevé que el sector de más rápido crecimiento será el de los implantes cardíacos, debido a los permanentes esfuerzos para mejorar el tratamiento y reducir el riesgo de mortalidad de las condiciones cardíacas. La demanda combinada de estos productos aumentará más del $14 \%$ anual, para llegar a 13.700 millones en 2007. Basado en la creciente evidencia clínica de la efectividad terapéutica, los seguros de salud están cubriendo un número creciente de pacientes con marcapasos, desfibriladores y Stents coronarios. Afirma que los progresos de la tecnología permiten a las personas afectadas con afecciones cardíacas vivir una vida más normal sin depender de complejos tratamientos farmacológicos.

MedSource Technologies es una empresa americana que cotizó en la bolsa neoyorquina entre 1999 y 2004 , y provee ingeniería, desarrollo de productos, servicios de manufactura y soluciones de gestión de la cadena de suministros a fabricantes de dispositivos médicos. Sus instalaciones en varios estados de USA y su filial en México sumaban unos $50.000 \mathrm{~m} 2$ y 1500 empleados. Sus ingresos durante el año fiscal 2003 ascendieron a casi U\$S 180 millones de dólares. El 30 de junio de 2004 la empresa fue adquirida por la corporación UTI, que de esta manera se convirtió en la empresa más grande de diseño, ingeniería, y manufactura por contrato que atiende al mercado de los dispositivos médicos.

Los grandes jugadores de los marcapasos:

- Medtronic

- Ventas: U\$S 9.000 millones en total, en el área de "Gestión del Ritmo Cardíaco" crecen al 30\% anual, poseen 32.000 empleados, y presencia en 120 países. Gastos en I+D de 800 millones de dólares anuales.

- Parte de Medtronic es el "Medtronic Microelectronic Center", Arizona, USA, fundado en 1973 y que actualmente ocupa a más de 1.400 empleados

- St Jude Medical's

- Ventas: U\$S 2.000 millones (43\% son marcapasos), casi U\$S 250 millones de I+D

- Guidant

- Ventas U\$S 3.700 millones (40\% desfibriladores, $18 \%$ marcapasos), 12.000 empleados. I+D son 500 millones

- Biotronik

- 2.500 personas, más del $60 \%$ dedicado a investigación, desarrollo o producción, ha vendido más de un millón de dispositivos implantables.

\section{Volviendo a los Marcapasos}

La existencia de empresas dedicadas a la investigación y desarrollo, en este caso en el área biomédica, está fuertemente asociada a la disponibilidad de financiamiento de riesgo para estos emprendimientos. Por tanto, existe una correlación fuerte entre el tamaño del mercado a que apunta la división ingeniería de CCC y la disponibilidad de capital de riesgo. Consecuentemente, luego del derrumbe del NASDAQ en 2000 (Ver Anexo 2), este mercado se ha contraído en forma importante. 
En respuesta a lo anterior, CCC se replantea su estrategia y en 2004 retoma el esfuerzo en la identificación activa de distribuidores (en los años anteriores, desde 1998, no se habían realizado esfuerzos sistemáticos en este sentido). Esta estrategia, más organizada y agresiva, está dando sus primeros resultados: está iniciando sus actividades un distribuidor en Turquía, y para 2005 está planeado el ingreso a Brasil, así como probablemente Perú y Paraguay,

Actualmente, CCC provee más del $35 \%$ del mercado interno y exporta a Argentina, Bahamas, Ecuador, Rusia, República Dominicana, Jamaica, India y Ucrania.

\section{Ingresar a Europa}

Existe una larga relación de CCC con el Centro Nacional de Microelectrónica (CNM) de España, y en particular con uno de sus investigadores líderes, Jordi Aguiló, quien ha estado en el Uruguay en reiteradas ocasiones. Es una persona muy amiga de América Latina, y de la integración de España y América Latina, y siempre ha estado vinculado a IBEROEKA y a IBERCHIP ${ }^{9}$, una iniciativa que surgió de él mismo. Aproximadamente en 1997 se presenta la posibilidad de iniciar proyectos de cooperación entre centros europeos y latinoamericanos con financiación de la Unión Europea y España. Recuerda Brum:

... el punto es que los proyectos tenían que ser multicéntricos, había que lograr la participación de muchos centros para que la propuesta fuera elegible. Nosotros desde CCC nos presentamos indicando nuestras capacidades, como quien tira una botella al mar, cuando además ni siquiera sabíamos exponer bien nuestras capacidades -hoy tenemos mucho más claro lo que sabemos y lo que no sabemos- una etapa muy primitiva. Nos presentamos, a ellos les pareció interesante, creo que fue en Brasil, a uno de esos encuentros sobre microelectrónica al que asistían las universidades y unas poquitas empresas. Y finalmente empezamos un proyecto conjunto, en el que participaron la Universidad de Lovaina, el CNM de España, un centro mexicano, la Universidad de los Andes (ULA) de Colombia y nosotros. El proyecto consistía en diseñar un dispositivo para tomar señales internas en el cuerpo y trasmitirlas al exterior a altas velocidades y capturarlas en una computadora. Un dispositivo que era muy interesante pero que en realidad no estaba muy claro que sirviera para nada, porque no había aplicaciones asociadas...

Como todas esas iniciativas que involucran a mucha gente, algunos trabajaron, otros no, o no lo hicieron bien. En esa interacción, la gente del CNM donde estaba Jordi trabajó muy bien, y nosotros también, y quedamos en una muy buena relación, porque los que sacamos adelante ese proyecto, que fue un éxito técnico pero nada más que eso, fuimos ellos y nosotros.

El CNM es un centro público que tiene sedes en Barcelona y en Sevilla. En Barcelona tiene sus instalaciones dentro del campus de la Universidad Autónoma de Barcelona, pero no depende de ella, aunque sí tiene profesores compartidos. El CNM de Sevilla se dedica a microcircuitos y el de Barcelona a la adquisición de señales. El CNM había fundado la empresa D+T, propiedad 50\% de $\mathrm{CNM}$, en consorcio con empresas tecnológicas de primera línea, como Lucent y Motorola. Estas empresas son socios minoritarios, integran el Board of Directors, y se involucran eventualmente en líneas de investigación del CNM. Si bien el emprendimiento no tuvo éxitos importantes, sobrevivió y en un momento dado surge el ofrecimiento de comenzar un emprendimiento conjuntamente con CCC.

Los uruguayos, por su parte, tenían gran interés en ingresar al mercado europeo, pero cada vez que realizaban presentaciones, asistían a eventos, ferias o visitaban empresas en el viejo continente, notaban que era muy difícil realizar negocios desde Uruguay, porque las empresas europeas son muy verticales, no están acostumbradas al outsourcing, y existen muchas trabas para los que no son europeos. Por esa razón los clientes de la división ingeniería de CCC están básicamente en Israel, Canada y USA y no en Europa. En grandes trazos, hay dos mercados: USA y Europa. Israel y Canada son subsidiarios del sistema americano. Por tanto, el desafío era ingresar al segundo mercado, Europa.

A D+T, por su parte, le interesaba una potencial alianza con CCC porque, si bien tenía capacidad en adquisición de señales, capacidad de realizar desarrollos en el ámbito médico, tenían serios problemas para finalizar los proyectos, problemas de gestión y de enfoque de proyectos. Se trataba de un grupo con un enfoque "muy académico", un grupo orientado a hacer la investigación pero no a terminarla. Tenían también problemas para cobrar y para conseguir clientes. Es así que ambas empresas identifican la oportunidad de asociarse, para complementar capacidades sinérgicamente:

Hicimos un acuerdo que se llama I2M, está en la web ${ }^{10}$. Además participa como socio minoritario de I2M una empresa colombiana, AGR-AIMS, que está vinculada a la Universidad de los Andes: $40 \%$ de D+T, $40 \%$ de CCC y $20 \%$ de los colombianos. Esto sucedió en el año 2000, en plena burbuja del Nasdaq, cuando existía una demanda voraz de microcircuitos y algunas empresas estaban tercerizando su diseño. Nos enteramos que ALCATEL estaba tercerizando parte de su diseño en Turquía, entonces resolvimos ofrecer nuestra capacidad de desarrollo de ASICs ${ }^{11}$.

Nosotros en CCC estábamos terminando con el $\mathrm{ASIC}^{12}$, en el que participó IBERCHIP en la fase de fabricación del integrado. Por otra parte, existían vínculos con ALCATEL. Existían tres empresas con sus respectivas "patas" universitarias: D+T tiene la pata universitaria del CNM, CCC tiene la pata universitaria de IIE con su capacidad del diseño de ASICs, y los colombianos tienen la pata universitaria de la ULA. Con ese esquema salimos a buscar maneras de funcionar, el tiempo fue pasando, y en el 2001 cuando ya todo el fervor del Nasdaq se estaba cayendo, armamos una gira por Europa, Jordi y yo, con fondos Europeos. Visitamos Paris, Barcelona, Madrid, Londres, Bruselas, visitamos varias empresas e institutos, pero el gran objetivo era ALCATEL. Cuando llegamos a ver a esa gente, nos dice que la demanda de diseño en microelectrónca está cayendo -ya lo sabíamos nosotros- están cerrando la operación en Turquía... habíamos llegado tarde. Ya se estaba desinflando toda la ola de la industria y empezaba a haber capacidad ociosa. Un año después, en el Silicon Valey había más desocupación que en el promedio de los Estados Unidos... agarramos la crisis del sector IT, vinculado con electrónica. Fue un intento en el medio de la caída. De todos modos, no perdimos nada, porque teníamos los subsidios europeos y la inversión había sido muy mínima: el local es del CNM, y el personal se limita a dos recursos a medio tiempo y si hay algún proyecto se contrata... pero mantuvimos el espíritu.

9 El proyecto IBERCHIP tiene por objetivo incrementar la cooperación entre los grupos industriales y académicos del área iberoamericana con actividad en el campo de la Microelectrónica. Es una red de servicio de fabricación de microsistemas para soporte de la industria y formación continua de expertos en microelectrónica. Está promovida y financiada por el Programa Iberoamericano de Ciencia y Tecnología para el Desarrollo (CYTED), enmarcado en el Área Temática de Tecnología de la Información y de las Comunicaciones. Véase www.iberchip.org.

10 http://www.i2m-design.com

11 Application-Specific Integrated Circuit, es decir, circuito integrado para aplicación específica. Se trata de un chip diseñado para una aplicación en particular (en oposición a los circuitos integrados de propósito general como la RAM o el procesador de una PC). Por lo general, los ASICs se construyen conectando bloques de circuitos existentes de una biblioteca. La existencia de estos "bloques prefabricados" torna más sencillo el diseño de ASICs que si se parte de cero.

12 Hace referencia al circuito diseñado para los marcapasos de CCC, que se describe más adelante. 
En los dos años sucesivos continuamos trabajando juntos, aunque se trató de años bastante oscuros, no resolvíamos nada, pero los subsidios europeos fueron lo suficientemente fuertes como para que no diéramos pérdida con esa estructura mínima. Participamos en congresos, nos involucramos en el desarrollo de un dispositivo con un grupo de investigación en Barcelona, un dispositivo estimulador de raíces sacras. El dispositivo ya estaba hecho, pero siempre hubo problemas con el electrodo, entonces el sistema nunca funcionó bien, hubo mil intentos de revivir eso, al final no revivió, y la vida más o menos siguió. Se hicieron cosas en el medio, cosas chicas.

Hoy (fines de 2004) estamos en un momento en el cual tenemos un proyecto concreto, es bien interesante, lo desarrollaron ellos en España y nosotros somos simplemente fabricantes: es un sensor de impedancia y temperatura para órganos que están siendo trasladados para transplantes... aparentemente uno de los problemas que hay para trasplantes es que el órgano no llega bien porque tuvo problemas con la temperatura o de otro tipo, que están vinculados con la impedancia, entonces aumenta la probabilidad de necrosis en ciertas zonas del órgano y lo van matando. El dispositivo hace posible poner una aguja en el órgano que se está trasladando, con unos sensores que envían información a un Palm directamente, y entonces cuando llega al centro de transplantes, leyendo en ese Palm está la historia de la impedancia y la temperatura durante todo el traslado. El sensor tiene todo un encapsulamiento de epoxy, una parte de mecánica biocompatible bastante complicada. En este momento, tenemos ese proyecto. Está en fase de prototipo, ya tiene un cliente, porque se comercializaron los derechos y hay una empresa que se llama Carburos que se va a encargar de vender eso en el mundo.

Esa es nuestra historia en España, que no es una historia exitosa. Tenemos una pequeña presencia, no perdimos; jugamos a la lotería y sacamos como para volver a comprar otro número... y este proyecto de los transplantes, bueno, es un proyecto muy interesante, da prestigio, pero nadie va a vivir de eso.

\section{Las alianzas dentro del país}

Es llamativo que, pese a la larga historia de CCC en el mercado uruguayo, no se visualice la aparición de un conjunto de empresas conexas con su actividad, es decir, que no se reconozca, ni siquiera en forma incipiente, un cluster en el área. Al profundizar en el tema, el primer punto a tener en cuenta es que, siendo éste un sector intensivo en conocimiento, son tanto o más importantes las alianzas con centros de $\mathrm{I}+\mathrm{D}$ que las tradicionales relaciones con proveedores de materias primas. Dentro de las áreas de investigación que naturalmente resultan de interés para la empresa está, por un lado, la Microelectrónica, y por otro, las áreas de la medicina que utilizan dispositivos implantables, en particular las vinculadas a la cardiología y a las neurociencias.

En Uruguay se desarrolló un Grupo de Microelectrónica (GME) en el Instituto de Ingeniería Eléctrica de la Facultad de Ingeniería de la Universidad de la República. En su momento (principios de los 90), la apuesta a esta área del conocimiento fue polémica, ya que no era obvio que pudieran surgir demandas locales para los resultados de la investigación y el desarrollo de ese grupo. Hoy el grupo se ha consolidado, y CCC considera de suma trascendencia su existencia. La vinculación formal del GME con CCC se produjo en 1994, con el objeto de desarrollar un ASIC para la línea de marcapasos de la empresa. Se trató de un proyecto de sustitución de híbridos, tecnología con la que CCC tuvo una mala experiencia, disparado por la necesidad de miniaturizar.

Como estrategia de gestión de riesgos, frente a esta tecnología novedosa para el país y para la empresa, CCC resolvió que el circuito integrado a desarrollar no tendría el microcontrolador incorporado. Ello se debió, por un lado, a que la capacidad de inversión era bastante acotada, y si se ponía el microcontrolador adentro del
ASIC, el mismo quedaba vinculado rígidamente a ese modelo de microcontrolador. Se decidió, por tanto, utilizar un poquito más de espacio y dejar el microcontrolador en el exterior del chip, lo que permite utilizar nuevos modelos de microcontroladores sin modificar el ASIC. Además, se evita el pago de royalties que hubiera significado incorporar el microcontrolador al ASIC de CCC. En síntesis, el ASIC resuelve la parte analógica del marcapasos, y funciona muy bien. La empresa comercializa dos modelos basados de marcapasos basados en ese ASIC: el TEROS 503 y el TEROS 603; además, está terminando para la primera mitad del año próximo los modelos bicamerales TEROS 703 y TEROS 803. La experiencia de colaboración con el grupo universitario, en opinión de Brum, es muy positiva:

Es bastante probable que en el correr del año que viene tengamos que embarquemos en hacer un segundo ASIC... el primer proyecto fue exitoso, tenemos ganas de repetir. Es cierto que no fue exitoso en los plazos que se pensaron originalmente, pero ello no se debió al grupo universitario, sino que fue culpa nuestra: fue justo la época en que nosotros dimos el "bandazo" hacia la venta de servicios de ingeniería, porque ahí estaba el mercado. Tuvimos el ASIC unos cuantos meses en un cajón. Primero tuvimos el diseño pronto y lo teníamos que validar, lo cual representaba una nueva inversión. Finalmente contratamos este servicio en Suiza, pero demoramos un poco en hacer esa inversión. Después había que diseñar todo lo que rodea al ASIC ... otra hubiera sido la historia si nosotros no hubiéramos tenido ese gran desarrollo por el lado de proyectos de ingeniería. Seguramente hubiéramos empezado el diseño del marcapasos en paralelo con la última etapa del ASIC, y el Tero 503, que entró al mercado en octubre de 2003, lo hubiera hecho un año y medio antes. El atraso no se debe entonces a problemas del ASIC, sino que es porque los marcapasos no estuvieron en nuestro foco en ese período de tiempo.

El segundo modelo de ASIC para CCC probablemente incorporará el microcontrolador. Estamos viendo que ya tenemos que arrancar el proyecto de desarrollo de la siguiente generación, porque estos proyectos demoran mucho tiempo.

Si bien en los hechos el único proyecto conjunto de envergadura que han realizado el GME y CCC es el ASIC para los marcapasos, la empresa ha intentado sistemáticamente comercializar los servicios del GME a los clientes de la división ingeniería. Cuenta Brum:

A todos nuestros clientes de ingeniería, en algún momento, le ofrecimos miniaturización... el tema es que los proyectos que nos han encomendado consumen muchísima energía, entonces el tamaño del dispositivo está dado por la batería y el tamaño del circuito no es un tema relevante. Por otra parte, los clientes saben que miniaturizar el circuito es un proyecto largo, costoso, etc. Pero no es que nuestros clientes hayan elegido servicios de miniaturización en otra parte, sino que no han miniaturizado, están sobre PCB. Siempre tenemos la esperanza y la opción de que el día que se pasen a ASICs vamos a poder venderles ese servicio y esa no es una capacidad de CCC, es una capacidad a la que CCC accede a través de su acuerdo estratégico con el GME de la Facultad.

En lo que respecta a la colaboración con grupos nacionales de investigación en el área médica, la realidad es distinta. CCC cuenta dentro de su staff con dos médicos y tiene una relación muy estrecha con el Instituto Nacional de Cirugía Cardiaca, pero este instituto no realiza investigación, y en consecuencia, no es un ámbito donde se estén generando sistemáticamente demandas por nuevos productos. En ese sentido, CCC tiene una relación fluida con la Fundación Favaloro, un reconocido instituto argentino de investigación con el que mantiene un permanente intercambio de ideas, lo que ha llevado a ambas organizaciones a considerar la realización de proyectos conjuntos que implicarían el diseño de nuevos productos por parte de CCC. Al interior de CCC existe una permanente tensión entre los desarrollos propios y los desarrollos para los clientes externos de la División Ingeniería, dado que los recursos a aplicar en ambos tipos de proyectos son los mismos. En la medida que se pusiera más el foco en los desarrollos propios, 
muy probablemente se concentrarían mucho más esfuerzos en la relación con la Fundación Favaloro, porque ello le permitiría a CCC sacar nuevos productos al mercado que hoy están "en carpeta", como por ejemplo el desfibrilador implantable. En relación a este producto, ya se han mantenido conversaciones para realizar toda la parte de ensayos con animales en dicha Fundación, que tiene muy buen bioterio. También el campo de la neuroestimulación es percibido por la empresa como muy promisorio, pero no existe en el país un grupo de neurólogos investigando en el área.

CCC no tiene mayores alianzas con empresas uruguayas del área médica, más allá de que, a igualdad de condiciones, prefiere proveedores locales, como lo demuestra la reciente sustitución de gabinetes de aluminio importados por otros construidos en Uruguay, al cambiar los precios relativos de los insumos. La casi inexistencia de estos acuerdos es una consecuencia de que prácticamente no existen proveedores nacionales en la cadena de valor de CCC, que sin embargo, observa con interés los movimientos que se producen en esa área, lo que por ejemplo le permitió identificar una empresa bastante pequeña que fabrica cables de grado médico para algunos de los dispositivos de CCC. En realidad, se trata de un ensamblador especializado: compra los insumos, arma los productos y estos salen con la marca CCC. Actualmente se explora la posibilidad de tercerizar con esa misma empresa el armado electrónico de dispositivos no implantables, dado que este proceso productivo no está en el foco de CCC.

\section{A la búsqueda de un buen nombre}

Nosotros tenemos un nombre muy poco feliz: Centro de Cardioestimuladores del Uruguay Sociedad Anónima. Si la telefonista de la empresa saludara con todo eso, quien llama se sentirá tentado a colgar antes de que termine la frase... se pierden negocios, etc. Entonces, a todos los efectos prácticos, el nombre siempre ha sido CCC.

Todos los dispositivos fabricados por CCC llevaban la inscripción CCC Uruguay, es decir, el nombre de la marca contenía la palabra Uruguay. Esto no es una práctica internacional. Por ejemplo, los cardioestimuladores de la competencia de CCC no dicen "Metronics USA" o "Biotronik Germany". La presencia del nombre del país corresponde más bien a un estado de ánimo de los fundadores de la empresa en un momento dado, que no es el de la actualidad ni tampoco se condice con la práctica común de todas las empresas. En consecuencia, hace ya algún tiempo se procedió a quitar la palabra "Uruguay" del logo, la empresa pasó a ser "solo" $\mathrm{CCC}$, en un proceso que necesariamente es lento y gradual, como relata Brum:

...yo todavía tengo tarjetas que dicen CCC Uruguay, que se diferencian claramente de mis tarjetas de I2M , que están en inglés, pese a ser una empresa de Barcelona. Mis nuevas tarjetas dicen solamente CCC. El nombre del país como parte de la marca no aportaba nada e inmediatamente generaba la pregunta Uruguay... ¿que es eso?. Hablar de Uruguay lo pone a uno en una situación incómoda, porque está connotado negativamente, simplemente porque como localización está fuera del circuito, es decir, este fenómeno no es algo específico de Uruguay: Pasa con cualquier país o región que no esté ni en Europa, ni en USA ni en oriente. Está fuera del ámbito. Claro que esto también es dinámico. Por ejemplo hoy la India "está en el ámbito", pero hace 5 años no lo estaba. Y en particular en el área médica no alcanza para una empresa siquiera decir que está en Estados Unidos... el "ámbito más selecto" está constituido por tres zonas: Boston, Miniápolis y California. Estar fuera de esas zonas es casi tan malo como estar en el Uruguay...

Entonces concurríamos a las ferias con nuestra cartelería utilizando solo las tres letras como marca, en particular a la feria denominada Medical Devices Manufacturers a la que vamos todos los años, por ser la más grande vinculada a los dispositivos médi- cos, donde asisten proveedores de outsourcing, gente que vende sistemas de calidad, gente que vende tornillos de titanio, o las cosas más extrañas. Por otro lado, nuestros afiches debían trasmitir la ingeniería, lo que ciertamente es difícil y utilizaban por ejemplo imágenes de las cajas de titanio de los dispositivos implantables. Entonces, muchas veces la gente se acercaba a preguntar ¿ustedes a qué se dedican... fabrican cajas de titanio? No era el caso. Por tanto, frente a la pregunta ¿ustedes que hacen?, nuestra respuesta es "nosotros hacemos dispositivos médicos". A raíz de esa experiencia, estamos comenzando a utilizar sistemáticamente la marca CCC medical devices.

\section{Anexo 1 Los certificados de calidad}

\section{Marcado CE}

El Marcado CE está regido por la Directiva 93/68/CEE de la Comunidad Europea y establece una serie de procedimientos de evaluación de la conformidad de los productos industriales con los objetivos o "requisitos esenciales" fijados por las directivas de armonización técnica, sobre todo en lo que respecta a la seguridad, la salud pública o la protección de los consumidores. Por otra parte, fija el régimen de colocación del marcado "CE" de conformidad en las directivas de armonización técnica sobre diseño, fabricación, comercialización, puesta en servicio o utilización de productos industriales.

La evaluación de la conformidad de los productos industriales corresponde a organismos designados por los Estados miembros, según el procedimiento comunitario de notificación de organismos. La lista de dichos organismos se publica en el Diario Oficial de las Comunidades Europeas. La evaluación de la conformidad se hace en general en dos etapas; una corresponde a la fase de diseño del producto y otra a su fase de fabricación. Se establecen ocho procedimientos de evaluación o módulos que se aplican a dichas fases de diversas maneras. Son los siguientes:

control interno de la fabricación

examen "CE" de tipo

conformidad con el tipo

aseguramiento de calidad de la producción

aseguramiento de calidad del producto

verificación de los productos

verificación por unidad

aseguramiento de calidad total.

Se autorizará el empleo de módulos adicionales o variaciones en la utilización de los módulos anteriormente mencionados, en caso de que las circunstancias específicas de un sector concreto o una directiva lo justifiquen. Si el procedimiento o los procedimientos de evaluación aplicados muestran que un producto concreto o un ejemplar representativo de la producción cumple los requisitos de la directiva particular que les sea aplicable, el fabricante o su mandatario establecido en la Comunidad Europea colocará el marcado "CE" en cada producto y hará una declaración escrita de conformidad. Este marcado:

tiene un único grafismo, que está formado por la sigla "CE" y el número de identificación del organismo notificado que interviene en la fase de control de la producción

se coloca en el propio producto, en el envase o en el documento de acompañamiento

permite la comercialización, la libre circulación y la utilización en la Comunidad del producto.

$\mathrm{Si}$ a un producto industrial se le aplican otras directivas que se ocupan de otros aspectos y establecen la colocación del marcado "CE", éste acreditará el cumplimiento de todas las directivas aplicables. Cualquier otra marca, especialmente las marcas de conformidad a normas naciona- 
les o europeas, podrá colocarse en productos industriales, salvo en caso de que pueda confundirse con el marcado "CE". Los Estados miembros impondrán sanciones a los fabricantes o a sus mandatarios en caso de que observen que el marcado "CE" se ha colocado indebidamente.

\section{Norma ISO 9001}

La Serie ISO 9000 es un conjunto de normas que en lugar de referirse al producto (su especificación, método de ensayo, método de muestreo, etc.) se refieren a la una forma de llevar a cabo la Gestión de la Calidad y montar los correspondientes Sistemas de la Calidad y Mejora Continua en una organización. En particular, la Norma ISO 9001, Sistemas de gestión de la calidad - Requisitos identifica los requisitos básicos del sistema de Gestión de la Calidad que resultan necesarios para garantizar que la organización cumpla determinados requerimientos y además posee prueba de ello, es decir se centra en proporcionar un producto satisfactorio a los clientes. Esta norma es la que se utiliza para la Certificación del Sistema.

\section{Norma ISO 13485}

La normal ISO 13485 (Oficialmente publicada en 2003), establece requerimientos directamente compatibles y bajo los cuales se puede implantar y certificar un sistema de gestión en la realización de productos y servicios en el sector médico. Son variantes de la Normativa Internacional ISO 9001. Los esquemas médicos ISO 13485, ISO 13488, FDA cGMP QSR ponen el acento en el factor de seguridad y efectividad de producto, primordialmente para administrar y controlar los procesos y actividades.

Los requisitos de seguridad y efectividad de producto prevalecen sobre el enfoque de calidad, centrado en las expectativas de cliente (paciente y familiares), aunque se puede aplicar dentro de un ámbito de mejora continua. Los requisitos regulatorios, específicamente del sector médico, se atienden y se cumplen bajo las normas ISO 13485 / ISO 13488.

\section{Anexo 2 \\ Variación del Mercado de Capital de Riesgo. “La Burbuja” del año 2000.}

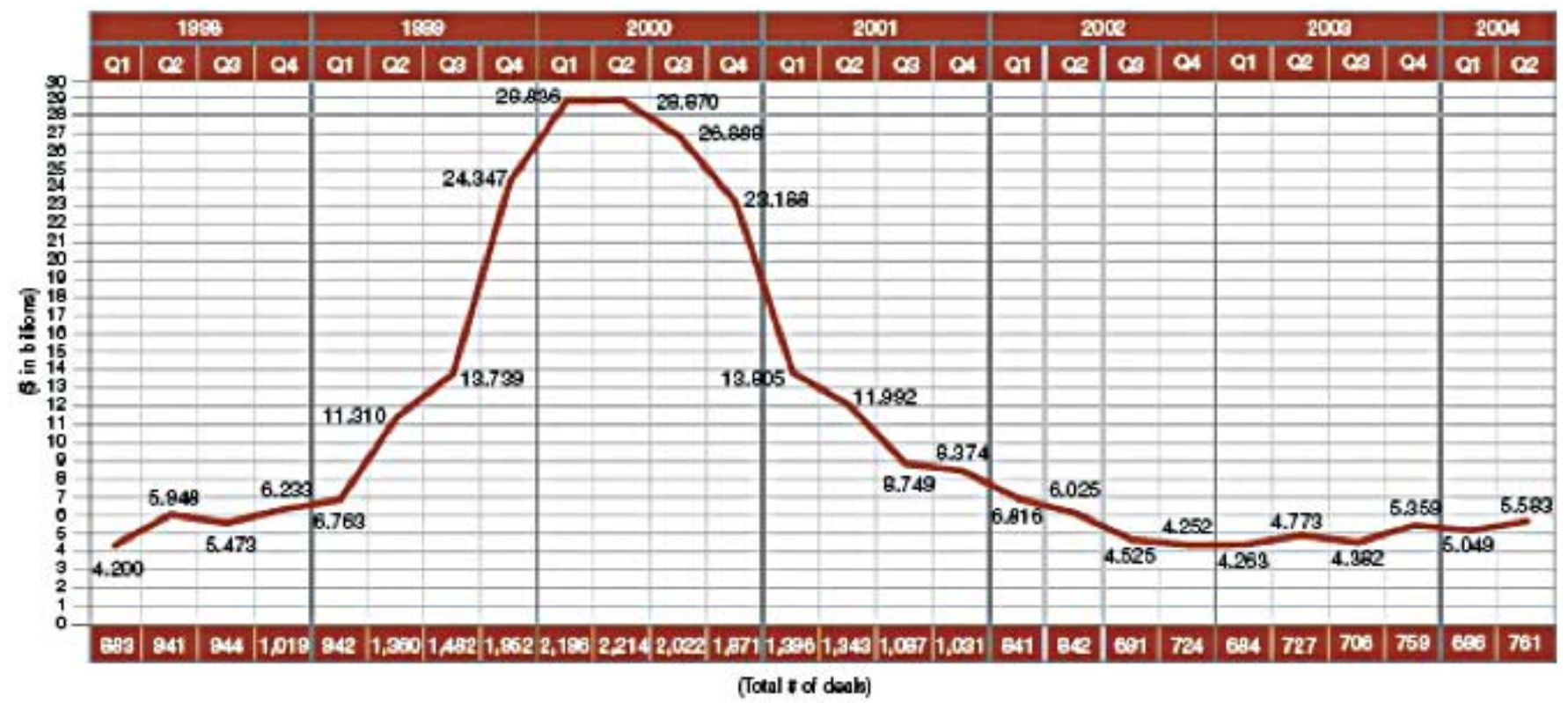

Fuente: http://www.pwcmoneytree.com/exhibits/04Q2MoneyTreeReport.pdf 